\title{
Addressing operational challenges of combatting malaria in a remote forest area of Vietnam using spatial decision support system approaches
}

\author{
Thang Duc Ngo, ${ }^{1}$ Sara E. Canavati, ${ }^{2}$ Ha Son Dinh, ${ }^{1}$ Thinh Duc Ngo, ${ }^{1}$ Duong T. Tran, ${ }^{1}$ \\ Nicholas J. Martin, ${ }^{3}$ Gerard C. Kelly ${ }^{2,4}$ \\ ${ }^{1}$ Department of Epidemiology, National Institute of Malariology, Parasitology and Entomology (NIMPE), \\ Hanoi, Vietnam; ${ }^{2}$ Vysnova Partners Inc., Hanoi, Vietnam; ${ }^{3}$ United States Naval Medical Research Unit Two, \\ Phnom Penh, Cambodia; ${ }^{4}$ Center for Biomedical Research, Burnet Institute, Melbourne, Australia
}

Correspondence: Gerard C. Kelly, Vysnova Partners Inc., Hanoi,
Vietnam. C/O U.S. Headquarters: Vysnova Partners Inc., 8400
Corporate Drive, Suite 130, Landover, MD 20785, USA.
Tel.+61.0.423.443.932
E-mail: gerardckelly@gmail.com

Key words: Malaria; Surveillance; Spatial decision support systems; Greater Mekong Subregion, Vietnam.

See online Appendix for additional figures.

Acknowledgements: the authors would like to thank the Vietnam National Institute of Malariology, Parasitology and Entomology (NIMPE) for their continued support and leadership. The team would also like to thank the hard-work and dedication of the Phu Yen Province Department of Health. In particular, the authors acknowledge the provincial malaria surveillance officers, Dong Xuan District malaria staff, commune heath station staff, and village health workers for their ongoing, tireless and dedicated work in supporting not only this research, but the national malaria program and overall elimination objectives of Vietnam.

Contributions: SC and GK provided equal contribution to this manuscript. The study was conceived and designed by GK and SC, with support from NM, HD, TN2, DT. Field research operations were coordinated by GK, TN1, HD. GK, NM, SC, TN2, DT guided scientific aspects of the study and provided detailed commentary on manuscript drafts. Data analysis and interpretation, and manuscript drafting were carried out by GK, SC, NM with support from all additional authors. All authors read and approved the final manuscript.

Conflict of interest: the authors declare no potential conflict of interest.

Funding: this work was supported by U.S. Navy Research and Development Funds Work Unit Number D1423.

Disclaimer: the views expressed in this article reflect the results of research conducted by the authors and do not necessarily reflect the official policy or position of the Department of the Navy, Department of Defense, nor the United States Government.

Received for publication: 28 February 2019.

Accepted for publication: 12 July 2019.

${ }^{\circ}$ Copyright: the Author(s), 2019

Licensee PAGEPress, Italy

Geospatial Health 2019; 14:770

doi:10.4081/gh.2019.770

This article is distributed under the terms of the Creative Commons Attribution Noncommercial License (CC BY-NC 4.0) which permits any noncommercial use, distribution, and reproduction in any medium, provided the original author(s) and source are credited.

\begin{abstract}
This study examines the development of a spatial decision support system (SDSS) to address operational challenges for combatting malaria in a priority remote forest area of Vietnam including locating active malaria transmission, guiding targeted response, and identifying mobile and high-risk populations. A customized SDSS was developed for three communes in Phu Yen Province, Vietnam. Geographical reconnaissance (GR) was conducted to map and enumerate all households in the study site. During 2015 and 2016, detected malaria cases were reported to the SDSS and georeferenced to household residence. Individual case data were analysed in the SDSS to guide targeted response. Case investigation data, including suspected forest and remote area transmission locations, were also integrated into the SDSS. Surveys and in-depth interviews were conducted to assess utility and user acceptability. In 2015 and 2016, 4,667 households and a population of 17,563 were captured during GR. During the study period, 128 malaria cases were reported and automatically mapped in the SDSS. Targeted response interventions were conducted in 12 villages, testing 1,872 individuals. Intervention and remote-area sleeping site data were mapped and analysed using the SDSS. During follow-up interviews in 2017 the SDSS was found to be highly acceptable to malaria surveillance personnel. Results suggest that an SDSS can provide an effective tool in Vietnam to support the implementation of specialized surveillance, and calls for further research, application and roll-out in the Greater Mekong Subregion.
\end{abstract}

\section{Introduction}

A key pillar of the World Health Organization's (WHO) Global Technical Strategy for Malaria 2016-2030 is the need to transform surveillance into a core intervention activity (WHO, 2015). In public health practice, the key objective of surveillance is to provide information to guide interventions and to empower decision makers to manage health programs more effectively (Nsubuga et al., 2006). This includes continuously and systematically capturing, interpreting and utilizing data to support all facets of operational management including planning, implementation and evaluation (WHO, 2006).

Health programs targeting malaria control or elimination require continuous access to data to determine the location of ongoing transmission and effectively support optimal delivery of 
appropriate interventions to relevant households and communities in need (GMAP, 2008; Moonen et al., 2010; Alonso et al., 2011; malERA Consultative Group on Monitoring, Evaluation, and Surveillance, 2011; WHO, 2012).

Key goals and opportunities for malaria surveillance, monitoring and evaluation in areas of low or moderation transmission include the need to identify population groups most at risk and to target interventions appropriately, ideally facilitated by mapping the areas of ongoing transmission (WHO, 2018). There can be major challenges to achieving such objectives however, particularly in the remote and resource-poor settings that often characterize the remaining malaria-endemic environments in the Greater Mekong Subregion (GMS).

Recent guidance from the WHO advise countries pursuing malaria control or elimination programs of the need to establish surveillance systems that are appropriate to the heterogeneous and changing nature of malaria epidemiology, particularly as transmission decreases within a country (WHO, 2018). Elements of an ideal information system to support effective malaria surveillance adopted in other countries include: rapid and complete case reporting, incorporation of data such as census or health survey information to support intervention delivery, automated and expert data analysis, customized outputs and feedback to guide targeted responses, and ideally, the integration of spatial mapping components (Ohrt et al., 2015). Surveillance needs can vary within a country in order to respond to increasing levels of subnational heterogeneity, whereby malaria transmission becomes increasingly localized to small, difficult to identify foci. Specialized tools, separate from larger national-level malaria surveillance or information systems, may be required to support the capture of relevant data in order to meet program objectives in such localized transmission areas. These tools however, should still maintain capacity to integrate and report relevant data into a centralized and broader routine health management information system (Ohrt et al., 2015; WHO, 2018).

Because the spatial distribution of malaria transmission is highly variable within endemic areas, map-based geo-spatial decision support tools can be used to identify and target interventions in an effective and efficient manner (malERA Consultative Group on Health Systems and Operational Research, 2011; Kelly et al., 2012, 2013; Clements et al., 2013). A spatial decision support system (SDSS) is a digital framework to facilitate decision making through relating routinely collected data to associated geographic locations, supporting spatial analysis and in the incorporation of customized algorithms, and generating automated reports and illustrative maps to target foci and manage interventions (Kelly et al., 2012). Figure 1 provides an illustration of a SDSS conceptual framework. These SDSSs, based on geographic information system (GIS) and global positing system (GPS) data capture and management technologies, can be used to integrate and map routinely collected programmatic and malaria surveillance data, together with associated census or health survey information including geographical reconnaissance (GR) data, to provide a suite of specialized operational tools supporting malaria program implementation (Kelly et al., 2012; Clements et al., 2013).

The Vietnam National Institute of Malariology, Parasitology, and Entomology (NIMPE) are currently pursuing an agenda of progressive malaria elimination, and between 1991 and 2014, the number of malaria cases and deaths in the country reduced by $97 \%$ and 99.8\% respectively (Thuan, 2001; NIMPE, 2011a, 2011b; Tran, 2015; UCSF, 2016). Despite the large reduction of the malar- ia burden in Vietnam, the country still faces significant challenges in managing and eliminating the disease, including: i) more than 15 million people residing in malaria-endemic areas; ii) remoteness of these malaria-endemic areas; iii) poor access to financial, human and other resources; iv) highly mobile populations (including cross-border movement); v) populations living and working in forests; and vi) drug-resistant malaria parasites (NIMPE, 2011a, 2011b; UCSF, 2016). Key objectives of Vietnam's strategy for malaria control and elimination include: i) improving access to early diagnosis; ii) prompt effective treatment; iii) ensuring uniform intervention coverage of at-risk populations; iv) improving the malaria epidemiological surveillance system; and v) ensuring sufficient resourcing and capacity for malaria epidemic response (NIMPE, 2011a, 2011b).

As transmission continues to decline in Vietnam, NIMPE are directing efforts to implement national objectives in priority, remote and hard-to-reach areas of persistent ongoing transmission, including forested and mountainous areas of central and south-central Vietnam (Erhart et al., 2005; Thanh et al., 2015). In support of Vietnam's pursuit of malaria elimination, SDSS based approaches were identified as potential supplementary surveillance-response tools to assist Vietnam meeting key objectives in these operationally challenging settings including: providing malaria personnel with a capacity to promptly report, map and track individual malaria infections; identify at risk-areas to target and implement associated responses; and incorporate household level census data to support the sufficient resourcing and optimal delivery of interventions. SDSS tools were selected in consideration of the specialized

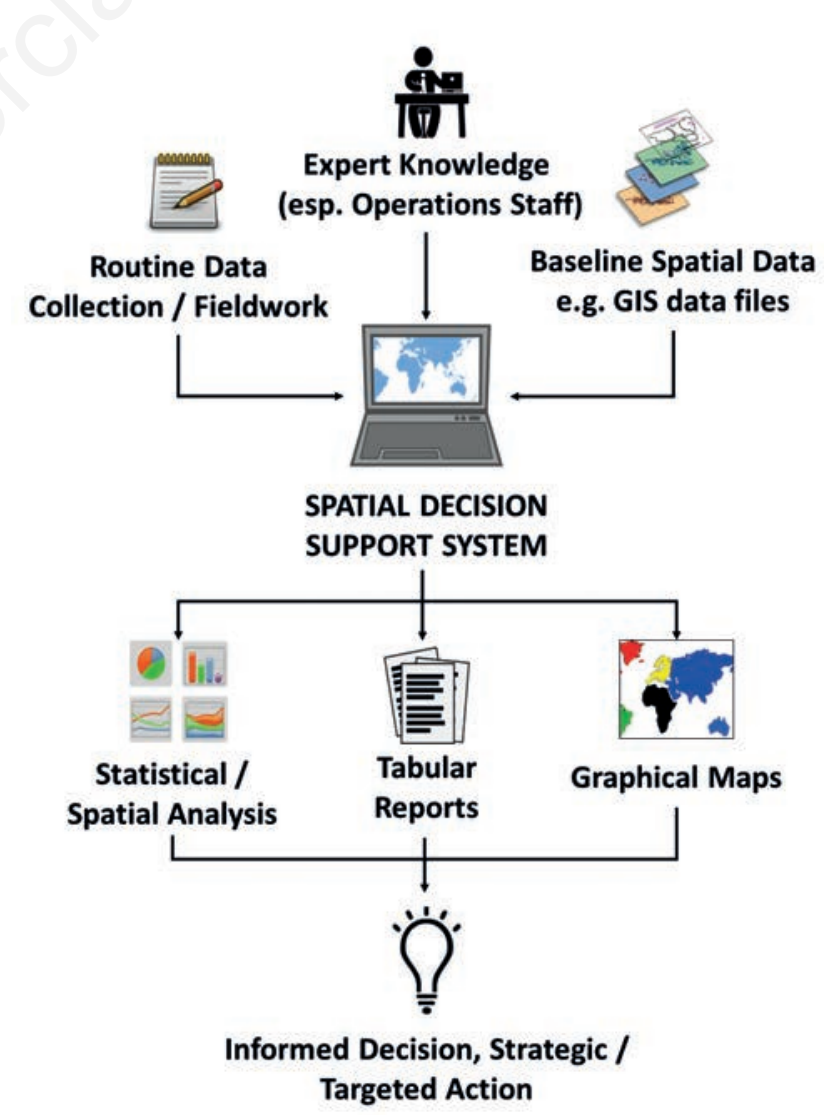

Figure 1. A spatial decision support system conceptual framework. GIS, geographic information system. 
surveillance needs in these priority settings, as well as the existing GIS operational capacity of the malaria program at both national and the targeted provincial level. Aims of this study were to develop and examine the effectiveness of customized SDSS based approaches to implement specialized malaria surveillance in a priority forest area of Vietnam. Objectives were to develop specialist surveillance tools to: automatically geo-locate passively reported malaria cases to the household level and guide targeted response interventions; and support the targeting and investigation of suspected forest transmission sites and identify associated population networks and interaction between forest-goers and their communities.

\section{Materials and Methods}

\section{Study area}

In 2014, a pilot SDSS for malaria surveillance was developed and established in the forested and mountainous district of Dong Xuan, Phu Yen Province, South Central Vietnam (Figure 2). Three communes were selected within Dong Xuan District as priority target sites to implement SDSS surveillance approaches: Phu Mo, Xuan Lanh, and Xuan Quang 1 (Figure 3). These communes were selected as they are directly located on the border of two Tier 1

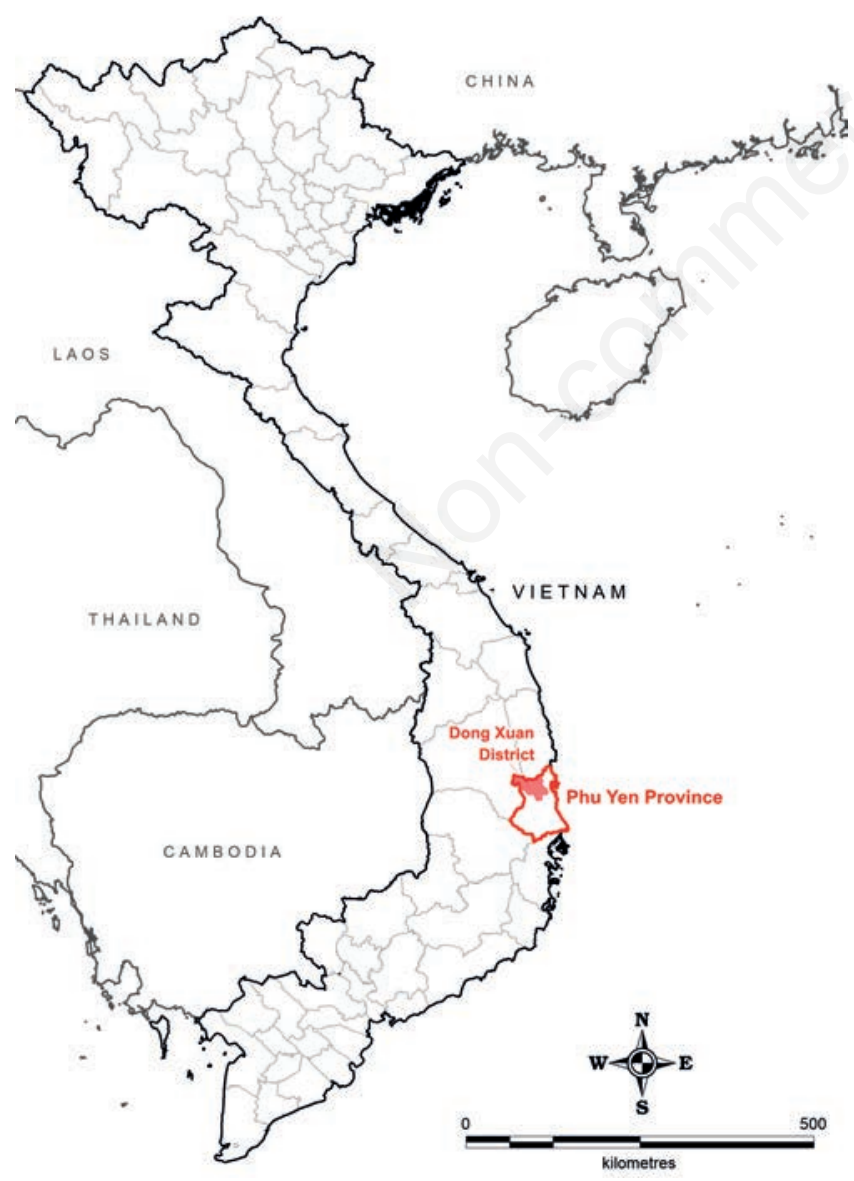

Figure 2. Location of Dong Xuan District, Phu Yen Province, Vietnam.
Artemisinin Resistant provinces as defined by the WHO Global plan for artemisinin resistance containment (WHO, 2014). Additionally, these sites have a high proportion of at-risk population that includes persons living and working on the forest fringe and within the forest. The study protocol was approved by the Vietnam Ministry of Health $(\mathrm{MoH})$ Institutional Review Board (IRB) under NIMPE Protocol number: 2016-VM-P1 and The University of California San Francisco Committee on Human Research (IRB \#: 14-13540 and Reference \#: 085636) in compliance with all applicable federal regulations governing the protection of human subjects. Additionally, NMRC-A Human Research Protection Official approved the study (HRPO.NMRCA. 2016.0002). Local authorities and community leaders were informed of the purpose and expected duration of the study.

\section{Development of a customized spatial decision support system}

A SDSS was developed using MapInfo Professional GIS and MapBasic programming (Pitney Bowes Inc., Stamford, Connecticut, USA) software. Baseline GIS data including topographic, demographic and administrative spatial layers were acquired from existing GIS data belonging to NIMPE and additional online free data sources, including the GADM database of Global Administrative Areas (www.gadm.org). Custom applications were developed using MapBasic and integrated these into the MapInfo Professional software to support the automation of standardized SDSS procedures designed to support enhanced malariasurveillance objectives.

Primary users of the SDSS were provincial-level malaria surveillance personnel from the Phu Yen Department of Health. Users of the SDSS were able to utilize both the customized SDSS applications as well as the full GIS software. The following customized applications to support the objectives of the study were developed: i) interactive baseline maps of the target SDSS site and greater Vietnam, ii) interactive household GR maps and associated attribute data, iii) automated malaria patient household residence mapping, iv) modules for targeting, planning, implementing and monitoring interventions, v) remote area sleeping site survey mapping, and vi) automatic household-level intervention coverage

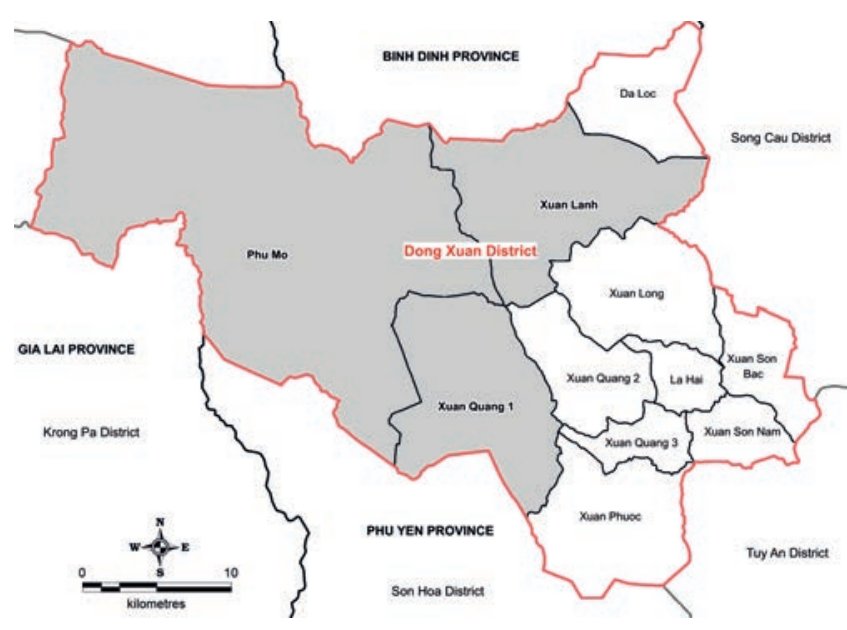

Figure 3. Location of study site communes, Dong Xuan District, Phu Yen Province, Vietnam. 
mapping, monitoring and assessment. Table 1 provides a summary of the specific custom SDSS applications that were developed to support surveillance operations as part of the study. Primary operation of the SDSS throughout the study was conducted by Phu Yen Province malaria surveillance personnel. Malaria case data from 2015 and 2016 were captured in the SDSS.

\section{Baseline data collection}

The research team conducted a one-day training programme in Dong Xuan District for village health workers (VHWs) from the target villages in the study who were selected to conduct baseline household GR operations. Teams of two VHWs worked together in their respective villages to collect household GR data during an 18-day period in January, 2015. Baseline household GR operations were conducted in all 18 villages located in the study communes. Trimble Juno T41 (Trimble Inc., Sunnyvale, CA) handheld computers with Arcpad software (ESRI, Redlands, CA), and smartphones (E4 model; Sony Corporation, Tokyo, Japan) with customized forms developed for data collection software (KLL Collect; Kathmandu Living Labs, Kathmandu, Nepal) were used for data collection. The following data were collected: date of survey, village name, unique household identification number, head of household name, and number of household residents. As part of field operations, all households mapped were also physically labelled on or near the front entrance of the house with a unique household identification number. Household GR data were uploaded into the SDSS, where interactive maps and both individual household level data and aggregate household and population data could be accessed by SDSS surveillance personnel.

\section{Rapid case reporting and investigation}

As per national guidelines, all malaria cases detected during the study were reported by phone to the Phu Yen provincial malaria office within 48 hours, which was followed by an investigation within three days (VNMOH, 2016). Malaria cases were recorded in an electronic spreadsheet (Microsoft Excel; Microsoft Corporation, Redmond, WA) by provincial SDSS surveillance personnel. Investigation data were also reported to provincial staff and updated in the spreadsheet. A MapBasic script was written for the GIS-based SDSS application to incorporate algorithms to automatically open, map and analyse the malaria case register data. This application enabled the surveillance-response officer to identify and select the household residence of the malaria patient and generate a user-defined response area radius. Surrounding households

Table 1. Summary of the custom applications developed as part of the Phu Yen province pilot malaria surveillance spatial decision support system.

General location topographic Incorporating census and survey and household mapping data to support intervention delivery

$\begin{array}{ll}\begin{array}{l}\text { Malaria patient household } \\ \text { residence mapping }\end{array} & \begin{array}{l}\text { Identification, mapping and tracking } \\ \text { of individual malaria infections and } \\ \text { ongoing transmission }\end{array}\end{array}$

A generic mapping tool with interactive functionality such as drawing, writing and illustration to rapidly develop custom operations maps to support programme activities and reporting

A spatial portal for accessing and querying individual and aggregate household level geographical reconnaissance data as required

Mapping of detected malaria cases to household residence location using automated applications to geo-reference rapidly reported patient information to household geographical reconnaissance location Interactive map-based interface to view the distribution of all reported malaria patient household residence locations and access individual patient level data Query-based dialog windows to develop customized malaria case maps based on user-defined parameters including date of diagnosis, malaria species and suspected mode of transmission

Household level targeted Identification and targeting interventions response to at-risk populations

Map-based interface allowing SDSS surveillance personnel to select malaria patient household locations and generate a user-defined response buffer radius to guide targeted interventions

Generation of summary data of total households and estimated population identified within designated response area to support intervention planning Automatic listing of neighbouring household identification data (e.g. household identification number, household name, population, etc.) to support the implementation and monitoring of target response interventions Automate generation of response area household level operation maps to support field operations

\begin{tabular}{lll}
$\begin{array}{l}\text { Remote area sleeping } \\
\text { site survey mapping } \\
\text { and analysis }\end{array}$ & $\begin{array}{l}\text { Identification, mapping and tracking } \\
\text { of at-risk populations }\end{array}$ & $\begin{array}{l}\text { Application to map remote area sleeping site survey location data collected as } \\
\text { part of routine field activities } \\
\text { Framework to develop population network maps to link remote area sleep site } \\
\text { members to their household residence locations }\end{array}$ \\
\hline $\begin{array}{l}\text { Intervention coverage } \\
\text { mapping, monitoring } \\
\text { and assessment }\end{array}$ & $\begin{array}{l}\text { Ensuring optimal delivery of } \\
\text { targeted interventions }\end{array}$ & $\begin{array}{l}\text { Application to automatically map household level targeted intervention } \\
\text { coverage data by status using a standardized data format } i . e . \text { household } \\
\text { receive intervention = yes, no, partial }\end{array}$ \\
& $\begin{array}{l}\text { Dialog widow to automatically generate intervention status summary } \\
\text { statistics and reports by user-defined administrative level } \\
(e . g . \text { village, commune, district, province) }\end{array}$ \\
\hline
\end{tabular}


were highlighted, automatically mapped, listed and ranked according to distance to the index household. Maps and household lists were automatically extracted and used to support field teams implementing the target response interventions.

\section{Remote area sleeping site survey assessments}

Remote-area sleeping site survey assessments were conducted in suspected non-village malaria transmission locations including forest, forest fringe and remote farming sites. Provincial-level SDSS malaria surveillance personnel utilized incoming malaria patient data to identify and select candidate sites of suspected malaria transmission occurrence in remote sleeping locations. Written informed consent was obtained from the selected candidates because some of the remote area sites are considered sensitive locations due to occupational activities conducted there. Teams collected the following data at these sites: location; description and assessment of working and sleeping conditions, identification of individuals at the site; case history of individuals; and potential mosquito breeding sites within the vicinity of the location. Data were collected using smart-phone mobile devices with data collection software and uploaded to the online database developed for this project (ONA; Ona Systems, Nairobi Kenya and Washington DC, USA) for storage, and data visualization. Data were also linked and accessed in the SDSS by provincial-level malaria surveillance personnel. Remote area sleeping site assessment data were mapped within the SDSS framework to create population network maps. The maps enabled linkage of key factors, including residences of index malaria cases identified through passive case detection, remote area sleeping sites where malaria transmission was suspected to have occurred, and the residential locations of identified remote area sleeping site members.

\section{Assessing user acceptability}

Structured, short-response user acceptability surveys and semistructured, in-depth interviews were conducted in March 2017 with SDSS stakeholders at national, provincial and sub-provincial levels to evaluate acceptability and perceived operational usefulness of the SDSS. A general user acceptability survey developed in English and translated into Vietnamese, was administered to 16 personnel and included a combination of closed and open-ended questions to identify user perceptions and highlight strengths, weaknesses and opportunities of SDSS-based malaria surveillance approaches. In-depth semi-structured interviews were also conducted with three key personnel who had direct involvement in the development and operation of the SDSS from national and provincial levels to gather further detailed qualitative data. Key themes explored as part of the semi-structured interviews included the strengths, weaknesses, challenges and opportunities of the SDSS, household GR operations, targeting of interventions using SDSS based approaches, and remote area sleeping site assessments.

\section{Results}

\section{Baseline household geographical reconnaissance}

A total of 4,667 households were mapped and enumerated during baseline GR surveys in Phu Mo, Xanh Lanh and Xanh Quang 1 target communes, covering a total of 17,563 people. Table 2 contains a breakdown of household and population data collected by village as part of the household GR. A screenshot of the GR user interface is provided as Appendix Figure A1.

\section{Malaria case detection and mapping}

In 2015 and 2016, 128 passively detected malaria cases were reported in Phu Mo (36), Xuan Lanh (45), and Xuan Quang 1 (47) communes, and registered in the SDSS. Of the 128 reported cases, 126 cases were successfully geo-referenced to their respective household residence locations and automatically mapped within the SDSS. The two cases that were not geo-referenced to household level were from patients residing outside of the three target communes where baseline GR data were not available. SDSS-generated malaria patient household residence maps for 2015 (Figure 4A) and 2016 (Figure 4B) are provided.

\section{Intervention targeting}

Targeted reactive case detection (RACD) interventions were conducted in 12 villages in the study communes between 2015 and 2016 , testing a total of 1,872 people. Zero additional malaria cases were detected during these activities. A screenshot of the SDSS user interface developed to support targeting village-level interventions is provided as Appendix Figure A2. Case investigation

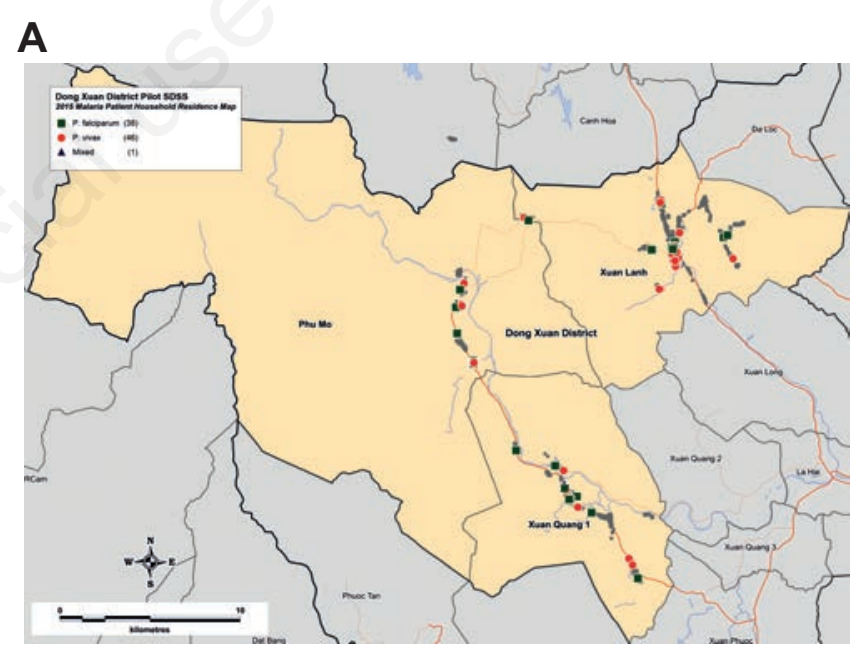

B

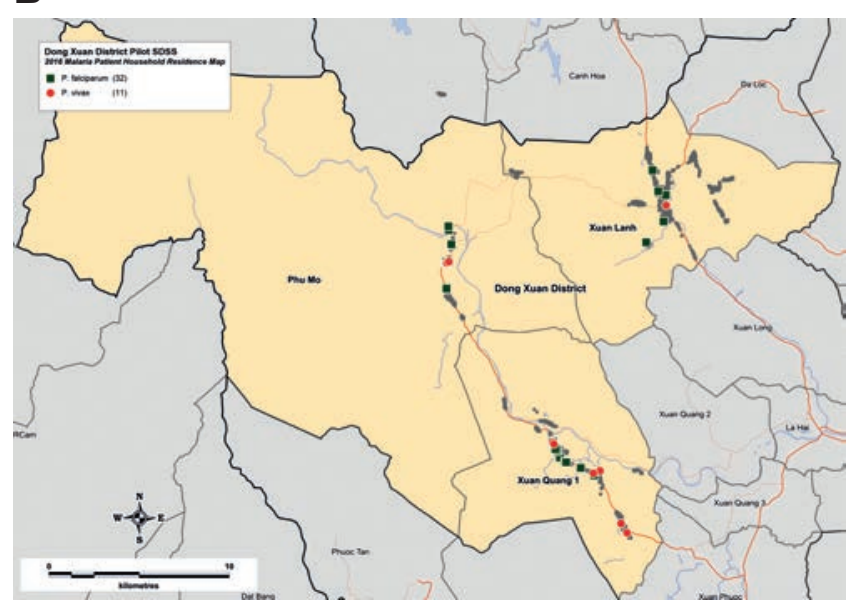

Figure 4. A) Spatial decision support system generated malaria patient household residence map, 2015; B) Spatial decision support system generated malaria patient household residence map, 2016. 
data were also assessed within the SDSS framework, including suspected mode of transmission and patient occupation data, to support the targeting and implementation of remote area sleeping site surveys. Table 3 provides a breakdown of detected malaria patient occupation data by commune between 2015 and 2016 .

\section{Remote area sleeping site assessments}

A total of 361 individuals were surveyed as part of remote area sleeping site assessments conducted within the SDSS target area in 2015 (51) and 2016 (310). Figure 5 illustrates a population network maps that links forest sleeping sites to permanent residence, allowing malaria surveillance personnel to target likely malaria exposure sites, follow up with patients at their household residence, and highlight potential communities at-risk of transmission.

\section{User acceptability}

User acceptability of the SDSS was high; with all questionnaire respondents $(16 / 16)$ indicating the SDSS was a useful surveillance tool and more effective than traditional approaches. Table 4 provides a breakdown of user acceptability survey respondent data.

Common themes highlighted during the user acceptability survey (16) and the semi-structured interviews (3) included: i) the strength of the SDSS in supporting surveillance, particularly in comparison to existing available tools; ii) the ability to detect places where malaria is occurring and to map malaria cases; iii) when the SDSS was applied, there was a more strategic approach and the teams knew where and what households needed to be reached; iv) a need to simplify and standardize data collection forms such as the remote area sleeping site assessment form; v) the use of GIS software allowed additional analysis, querying and use of other data to be independently conducted as desired outside of a locked framework; and vi) a need for the continued provision of training, technical support and funding for the SDSS.

\section{Discussion}

Currently, the Vietnam Malaria Information System does not support nationwide case-based reporting and faces obstacles adequately supporting key elements of elimination such as early detection of transmission foci and targeting response (WPRO, 2018). Results from the study indicate technical capacity of the SDSS to support specialist surveillance implementation based on malaria case distribution maps (Figures 4 and 5), data summaries (Table 2) and patient occupation analysis (Table 3) in selected priority areas. Results also revealed strong programme support and operational capacity for implementing SDSS-based surveillance approaches. The high case geo-reference rate $(100 \%$ of possible cases) reflected in the results is indicative of the strong organization within the Vietnam Malaria programme and sound communication channels existing between operational levels, from village level through to provincial and national. Given the GIS technical capacity at both national and provincial levels, SDSS-based surveillance operations remain ongoing. These operational qualities provide favourable conditions to apply SDSS based approaches to support specialized surveillance interventions in localized settings, such as priority malaria endemic communes or districts. As indicated by the user acceptability qualitative results, the SDSS was perceived as an effective and more efficient tool to support

such applications, including locating and mapping cases both in village and forest settings, and targeting and implementing associated response interventions, but was also limited by funding, resources, and additional training support.

As Appendix Figure A2 illustrates, a key strength of the SDSS is the interactivity of the user interface.

Table 2. Summary of household geographical reconnaissance data collected in Dong Xuan District, Phu Yen Province, Vietnam, 2015.

\begin{tabular}{lccc} 
Commune & Village & Households & Population \\
Phu Mo & Phu Dong & 142 & 574 \\
& Phu Giang & 242 & 944 \\
& Phu Hai & 61 & 288 \\
& Phu Loi & 102 & 391 \\
& Phu Tien 123 & 236 & 932 \\
Xuan Lanh & Phu Mo Total & 783 & 3129 \\
& Da Du & 343 & 1385 \\
& Ha Rai & 245 & 927 \\
& Lanh Cao & 112 & 436 \\
& Lanh Truong & 366 & 1313 \\
& Lanh Tu & 114 & 426 \\
& Lanh Van & 935 & 3450 \\
& Soi Nga & 224 & 825 \\
& Xi Thoai & 192 & 670 \\
& Xuan Lanh Total & 2531 & 9432 \\
\hline Xuan Quang 1 & Dong Hoi & 70 & 254 \\
& Ky Lo & 480 & 1778 \\
& Phu Tam & 160 & 631 \\
& Suoi Coi 1 & 300 & 1050 \\
& Suoi Coi 2 & 343 & 1379 \\
& Xuan Quang 1 Total & 1353 & 5092 \\
& Total & 4667 & 17653 \\
\hline
\end{tabular}

Table 3. Summary of detected malaria patient occupations by commune between 2015 and 2016.

$\begin{array}{lccc}\text { Patient } & \text { Phu Mo } & \text { Xuan Lanh } & \text { Xuan Quang } 1 \\ \text { occupation } & \% \text { (n) } & \% \text { (n) } & \% \text { (n) } \\ 2015-2016 & & & \end{array}$

Work in farm $\quad 25 \quad 0 \quad 2$

\begin{tabular}{cccc} 
& $(9)$ & $(0)$ & $(1)$ \\
Timber harvesting & 31 & 9 & 47 \\
& $(11)$ & $(4)$ & $(22)$ \\
\hline
\end{tabular}

\begin{tabular}{llll}
\hline Plantation worker & 33 & 51 & 40
\end{tabular}

$(12)$

\begin{tabular}{lcc} 
Local resident & 11 \\
& $(4)$ & $(0)$ \\
\hline Aloe wood collection & 0
\end{tabular}

Charcoal

\begin{tabular}{cccc} 
& $(0)$ & $(1)$ & $(0)$ \\
Not stated & 0 & 0 & 2 \\
& $(0)$ & $(0)$ & $(1)$ \\
\hline
\end{tabular}




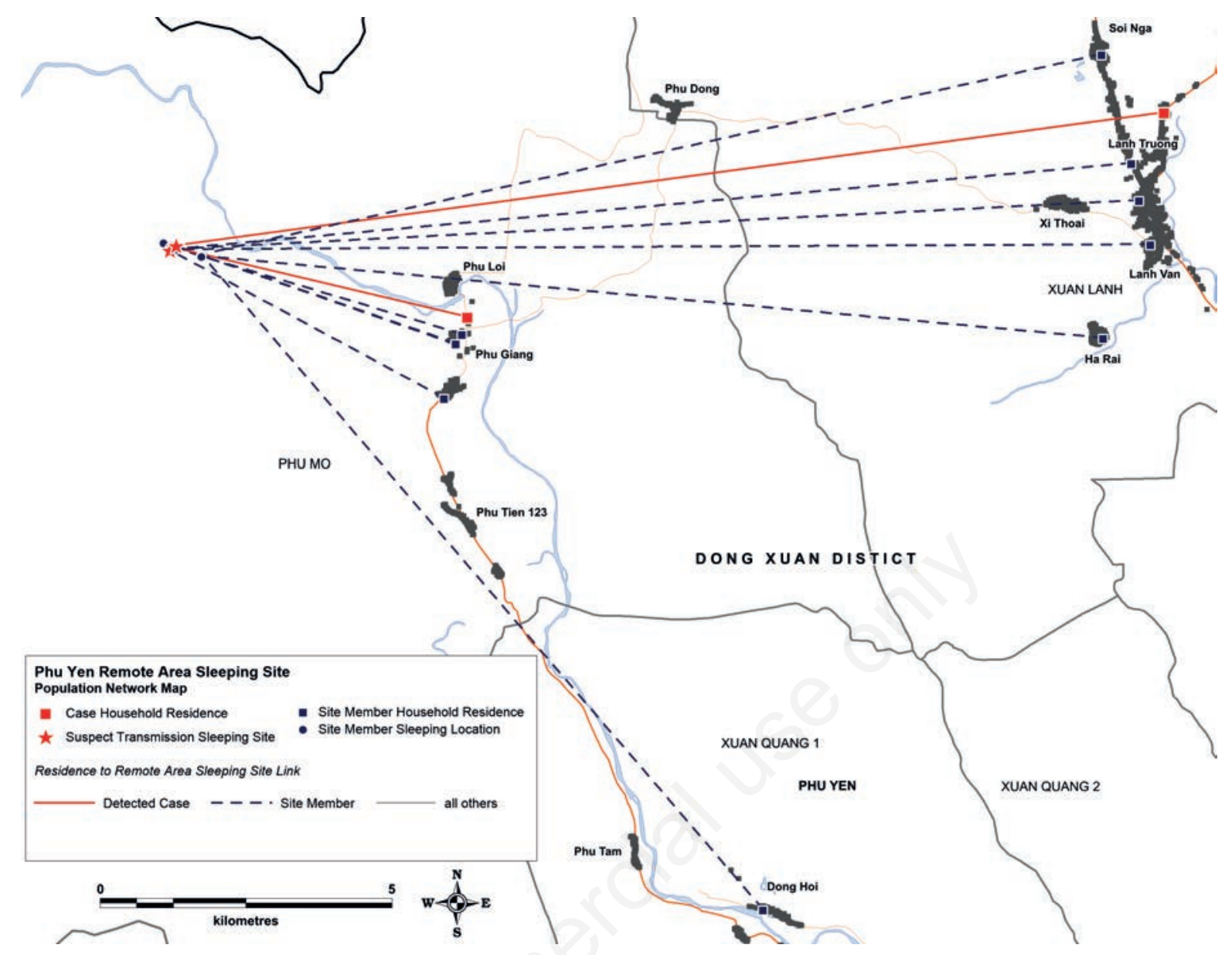

Figure 5. Example of a spatial decision support system generated population networks map linking remote area sleeping sites with detected malaria cases to permanent residences of sleeping site members to support follow-up interventions.

Table 4. Phu Yen province pilot malaria surveillance spatial decision support system user acceptability survey response data.

\begin{tabular}{|c|c|c|c|c|c|}
\hline Survey question & $\begin{array}{l}\text { Strongly agree } \\
\% \text { (n) }\end{array}$ & $\begin{array}{l}\text { Agree } \\
\%(n)\end{array}$ & $\begin{array}{l}\text { Undecided } \\
\% \text { (n) }\end{array}$ & $\begin{array}{l}\text { Disagree } \\
\% \text { (n) }\end{array}$ & $\begin{array}{l}\text { Strongly disagree } \\
\% \text { (n) }\end{array}$ \\
\hline $\begin{array}{l}\text { The SDSS provides a useful tool for locating malaria } \\
\text { cases on a map }\end{array}$ & $\begin{array}{l}43.75 \\
(7)\end{array}$ & $\begin{array}{l}56.25 \\
(9)\end{array}$ & $\begin{array}{c}0.00 \\
(0)\end{array}$ & $\begin{array}{l}0.00 \\
(0)\end{array}$ & $\begin{array}{l}0.00 \\
(0)\end{array}$ \\
\hline The SDSS provides a useful tool for locating households on a map & $\begin{array}{l}43.75 \\
(7)\end{array}$ & $\begin{array}{l}56.25 \\
(9)\end{array}$ & $\begin{array}{l}0.00 \\
(0)\end{array}$ & $\begin{array}{l}0.00 \\
(0)\end{array}$ & $\begin{array}{l}0.00 \\
(0)\end{array}$ \\
\hline $\begin{array}{l}\text { The SDSS provides a useful tool for identifying where malaria } \\
\text { transmission and potential outbreaks are occurring }\end{array}$ & $\begin{array}{c}50.00 \\
(8)\end{array}$ & $\begin{array}{c}43.75 \\
(7)\end{array}$ & $\begin{array}{l}6.25 \\
(1)\end{array}$ & $\begin{array}{l}0.00 \\
(0)\end{array}$ & $\begin{array}{l}0.00 \\
(0)\end{array}$ \\
\hline $\begin{array}{l}\text { The SDSS provides a useful tool for planning where } \\
\text { to conduct malaria response interventions }\end{array}$ & $\begin{array}{l}31.25 \\
(5)\end{array}$ & $\begin{array}{l}68.75 \\
(11)\end{array}$ & $\begin{array}{l}0.00 \\
(0)\end{array}$ & $\begin{array}{l}0.00 \\
(0)\end{array}$ & $\begin{array}{l}0.00 \\
(0)\end{array}$ \\
\hline $\begin{array}{l}\text { The SDSS provides a useful tool for monitoring and evaluation } \\
\text { malaria response intervention coverage and progress }\end{array}$ & $\begin{array}{c}37.50 \\
(6)\end{array}$ & $\begin{array}{l}62.50 \\
(10)\end{array}$ & $\begin{array}{l}0.00 \\
(0)\end{array}$ & $\begin{array}{l}0.00 \\
(0)\end{array}$ & $\begin{array}{l}0.00 \\
(0)\end{array}$ \\
\hline $\begin{array}{l}\text { The SDSS is useful for generating malaria surveillance } \\
\text { maps and reports }\end{array}$ & $\begin{array}{c}56.25 \\
(9)\end{array}$ & $\begin{array}{c}43.75 \\
(7)\end{array}$ & $\begin{array}{l}0.00 \\
(0)\end{array}$ & $\begin{array}{l}0.00 \\
(0)\end{array}$ & $\begin{array}{l}0.00 \\
(0)\end{array}$ \\
\hline $\begin{array}{l}\text { The SDSS and mapping applications were easy } \\
\text { to understand and use }\end{array}$ & $\begin{array}{c}25.00 \\
(4)\end{array}$ & $\begin{array}{c}62.50 \\
(10)\end{array}$ & $\begin{array}{l}12.50 \\
(2)\end{array}$ & $\begin{array}{l}0.00 \\
(0)\end{array}$ & $\begin{array}{l}0.00 \\
(0)\end{array}$ \\
\hline $\begin{array}{l}\text { SDSS Household and Remote-Area maps are a useful tool } \\
\text { to support surveillance-response activities }\end{array}$ & $\begin{array}{c}25.00 \\
(4)\end{array}$ & $\begin{array}{l}75.00 \\
(12)\end{array}$ & $\begin{array}{l}0.00 \\
(0)\end{array}$ & $\begin{array}{l}0.00 \\
(0)\end{array}$ & $\begin{array}{l}0.00 \\
(0)\end{array}$ \\
\hline SDSS maps are easy to interpret in the field & $\begin{array}{c}18.75 \\
(3)\end{array}$ & $\begin{array}{l}68.75 \\
(11)\end{array}$ & $\begin{array}{l}6.25 \\
(1)\end{array}$ & $\begin{array}{l}6.25 \\
(1)\end{array}$ & $\begin{array}{l}0.00 \\
(0)\end{array}$ \\
\hline $\begin{array}{l}\text { The SDSS is a more effective tool to support } \\
\text { surveillance-response than traditional approaches }\end{array}$ & $\begin{array}{c}31.25 \\
(5)\end{array}$ & $\begin{array}{c}68.75 \\
(11)\end{array}$ & $\begin{array}{l}0.00 \\
(0)\end{array}$ & $\begin{array}{l}0.00 \\
(0)\end{array}$ & $\begin{array}{l}0.00 \\
(0)\end{array}$ \\
\hline
\end{tabular}

SDSS, spatial decision support system. 
This allowed for malaria surveillance personnel to actively view and search the distribution of detected malaria cases, independently select target areas around cases where response is required, and to extract relevant household-level data to support operations. As previous studies have demonstrated, a SDSS framework can be automated to generate target response areas based on user-defined parameters within specific geographic regions and transmission foci classifications (Kelly et al., 2013). These automated response areas can also be interactively revised by the SDSS-user as deemed necessary; determined by an array of factors including current caseloads within the target area, local complexities or additional factors that may influence transmission (such as mobile populations), national surveillance policies and guidelines, or the resources available to the programme.

In the context of the GMS, key results from the study included the capacity to integrate remote forest area sleeping site assessment data into the SDSS platform, the first time these data have been mapped and utilized within an SDSS. As reflected in Figure 5 , this tool provided malaria surveillance personnel the ability to develop network maps of malaria patients passively detected at a clinic level, linking their household residence location to the suspected remote area transmission site, and identifying additional individuals sleeping in the same remote area back to their respective household residences. The lack of malaria cases detected during village based RACD activities suggests that these network maps and remote transmission sites may be critical to identifying additional cases likely linked by occupation in the forest.

Whilst technical skills in GIS operation is not a pre-requisite for SDSS operation, additional functionality associated with housing an SDSS within an open GIS software has the potential to further facilitate the role of a health surveillance officer operation to conduct specialized epidemiological investigation and guide decision making, and was demonstrated in this study. As illustrated in Figure 1, a key element of any SDSS is expert knowledge of the user (Kelly et al., 2012). In complex epidemiological situations such as those associated with forest malaria and highly mobile populations, input via expert decision makers into the surveillanceresponse process is essential (i.e. the input of local level surveillance personnel). Providing surveillance personnel access to flexible tools such as an SDSS in addition to larger routine off-the-shelf national level information or surveillance systems, which are generally and understandably less interactive or flexible, can be advantageous. This includes running customized geo-spatial queries, overlaying additional ad hoc data, and manipulating spatial data layers such as target response area radii as required. When integrated into a broader health information or surveillance system, providing a capacity to pull relevant data such as individual malaria cases into an associated offline SDSS has the opportunity to provide an enhanced decision support tool to implement specialized surveillance in priority locations without threatening data quality. Given the high level of heterogeneity that can occur in the transmission of malaria sub-nationally, SDSS tools could supplement national surveillance or information systems in selected areas.

Limitations of this study included the inability of the SDSS to map malaria cases or identified high risk individuals to their household residencies if they were located outside of the study area. Whilst baseline household GR operations are somewhat resource intensive experiences from malaria surveillance personnel and village health workers utilizing these data in Vietnam proved favourable. Minimal training investment and external technical oversight was provided in Phu Yen and, as reflected in Table
2 , a relatively large number of households $(4,667)$ and people $(17,653)$ were captured over the 18 days of data collection. Given the ever-increasing availability, familiarity, and accessibility of mobile smart phone devices, even in remote areas, this study indicates the feasibility of implementing household level GR data collection in priority areas, particularly if programmes have strong community-level personnel such as village health workers or malaria surveillance officers. Opportunities to integrate with other health and household-based community programmes or utilize external census data could also be explored as alternatives to sourcing baseline GR data. Ideally, malaria elimination interventions should be monitored at high resolution where possible due to micro-epidemiological variations in transmission, such as a household or remote-area sleeping site level; however SDSS-based approaches are capable of utilizing lower-resolution baseline data as operationally feasible, such as village, commune, forest, and district-level data (Bousema et al., 2012). This study also did not evaluate costs of establishing the SDSS given the existence of GIS software within the malaria programme and GIS operational skillsets of SDSS-users. Costing studies have been conducted regarding development and implementation of SDSS in the Pacific (Marston et al., 2014), however similar studies relevant to the GMS would be beneficial.

Moving forward, there is a need for further standardization, streamlining, and optimization of the SDSS-based surveillance approaches piloted in this study to more efficiently capture essential information and utilize routinely reported data. This study focused on the application of SDSS for malaria case identification; however, the same approach could be applied to other vector-borne disease or community-based health programmes that would benefit from geo-spatial surveillance and management tools. Improved information coupled with GPS data can strengthen decision making and guide deployment of targeted interventions. Opportunities exist to utilize internet and mobile reporting device technologies to improve current phone-based case reporting and field data collection. Options for web-based approaches to SDSS analysis and data dissemination should also be explored.

\section{Conclusions}

This study presents an approach to enhanced malaria surveillance in the GMS using SDSS technology, the first of its kind within this epidemiologically significant region. Results from this study demonstrated that SDSS approaches can support the implementation of specialized surveillance in priority forest areas. This approach moves beyond the routine reporting and display of malaria data to providing interactive geo-spatial decision support tools to facilitate active surveillance operations and investigation. Utilizing these tools enabled malaria programme personnel to locate malaria transmission locations in near real time. The application of acceptable high-resolution surveillance tools with the capability to target interventions is useful for malaria programme personnel at the implementation level of operations. Promising findings from this pilot study call for expanded operational research to further measure and quantify impacts, standardization, and potential to scale SDSS approaches in Vietnam and the wider GMS. 


\section{References}

Alonso PL, Brown G, Arevalo-Herrera M, Binka F, Chitnis C, Collins F, Doumbo OK, Greenwood B, Hall BF, Levine MM, Mendis K, Newman RD, Plowe CV, Rodriguez MH, Sinden R, Slutsker L, Tanner M, 2011. A research agenda to underpin malaria eradication. PLoS Med 8:e1000406.

Bousema T, Griffin JT, Sauerwein RW, Smith DL, Churcher TS, Takken W, Ghani A, Drakeley C, Gosling R, 2012. Hitting hotspots: spatial targeting of malaria for control and elimination. PLoS Med 9:e1001165.

Clements ACA, Reid HL, Kelly GC, Hay SI, 2013. Further shrinking the malaria map: how can geospatial science help to achieve malaria elimination? Lancet Infect Dis 13:709-18.

Erhart A, Ngo DT, Phan VK, Ta TT, Van Overmeir C, Speybroeck N, Obsomer V, Le XH, Le KT, Coosemans M, D'alessandro U, 2005. Epidemiology of forest malaria in central Vietnam: a large scale cross-sectional survey. Malaria J 4:58.

GMAP, 2008. The global malaria action plan. Roll Back Malaria Partnership, World Health Organization, Geneva, Switzerland.

Kelly G, Hale E, Donald W, Batarii W, Bugoro H, Nausien J, Smale J, Palmer K, Bobogare A, Taleo G, Vallely A, Tanner M, Vestergaard L, Clements A, 2013. A high-resolution geospatial surveillance-response system for malaria elimination in Solomon Islands and Vanuatu. Malaria J 12:108.

Kelly GC, Tanner M, Vallely A, Clements A, 2012. Malaria elimination: moving forward with spatial decision support systems. Trends Parasitol 28:297-304.

malERA Consultative Group on Health Systems and Operational Research, 2011. A research agenda for malaria eradication: health systems and operational research. PLoS Med 8:e1000397.

malERA Consultative Group on Monitoring, Evaluation, and Surveillance, 2011. A research agenda for malaria eradication: monitoring, evaluation, and surveillance. PLoS Med 8:e1000400.

Marston L, Kelly G, Hale E, Clements A, Hodge A, Jimenez-Soto E, 2014. Cost analysis of the development and implementation of a spatial decision support system for malaria elimination in Solomon Islands. Malaria J 13:325.

Moonen B, Cohen JM, Snow RW, Slutsker L, Drakeley C, Smith DL, Abeyasinghe RR, Rodriguez MH, Maharaj R, Tanner M, Targett G, 2010. Operational strategies to achieve and maintain malaria elimination. Lancet 376:1592-603.

NIMPE, 2011a. National strategy for malaria control and elimination for the period of 2012-2015. National Institute of Malariology, Parasitology and Entomology, Hanoi, Vietnam.

NIMPE, 2011b. National strategy for malaria control and elimination in the period 2011-2020 and orientation to 2030. National
Institute of Malariology, Parasitology and Entomology, Hanoi, Vietnam.

Nsubuga P, White ME, Thacker SB, Anderson MA, Blount SB, Broome CV, Chiller TM, Espitia V, Imtiaz R, Sosin D, Stroup DF, Tauxe RV, Vijayaraghavan M, Trostle M, 2006. Public health surveillance: a tool for targeting and monitoring interventions. In: Jamison DT, Breman JG, Measham AR, Alleyne G, Claeson M, Evans DB, Jha P, Mills A, Musgrove P, eds. Disease control priorities in developing countries. 2nd ed. World Bank, Washington DC, pp 997-1015.

Ohrt C, Roberts KW, Sturrock HJW, Wegbreit J, Lee BY, Gosling $\mathrm{RD}, 2015$. Information systems to support surveillance for malaria elimination. Am J Trop Med Hyg 93:145-52.

Thanh PV, Van Hong N, Van Van N, Van Malderen C, Obsomer V, Rosanas-Urgell A, Grietens KP, Xa NX, Bancone G, Chowwiwat N, Duong TT, D'Alessandro U, Speybroeck N, Erhart A, 2015. Epidemiology of forest malaria in Central Vietnam: the hidden parasite reservoir. Malaria J 14:86.

Thuan LK, 2001. Scientific work 2001-2015. Medical Publishing House, National Institute of Malariology, Parasitology and Entomology, Hanoi, Vietnam.

Tran DT, 2015. Final report of the national malaria program 2014 and plan for 2015 in Vietnam. National Institute for Malariology, Parasitology and Entomology, Hanoi, Vietnam.

UCSF, 2016. APMEN country briefing: elimination malaria in Vietnam. Malaria Elimination Initiative, Global Health Group, University of California, San Francisco.

VNMOH, 2016. Guideline of malaria monitoring and preventing. Vietnam Ministry of Health, Hanoi, Vietnam.

WHO, 2006. Communicable disease surveillance and response systems - Guide to monitoring and evaluating. World Health Organization, Geneva, Switzerland.

WHO, 2012. Disease surveillance for malaria elimination. An operational manual. Roll Back Malaria Partnership, World Health Organization, Geneva, Switzerland.

WHO, 2014. Status report on artemisinin resistance, World Health Organization Global Malaria Programme. World Health Organization, Geneva, Switzerland.

WHO, 2015. Global technical strategy for malaria 2016-2030. Geneva, World Health Organization.

WHO, 2018. Malaria surveillance, monitoring \& evaluation: a reference manual. World Health Organization, Geneva, Switzerland.

WPRO, 2018. National malaria programme review - Viet Nam. World Health Organization Regional Office for the Western Pacific, Manila, Philippines. 